\title{
Editorial
}

\section{Suicide at Very Advanced Age - The Extremes of the Gender Paradox}

\author{
Diego De Leo and Kairi Kõlves
}

Australian Institute for Suicide Research and Prevention, Griffith University, Brisbane, QLD, Australia

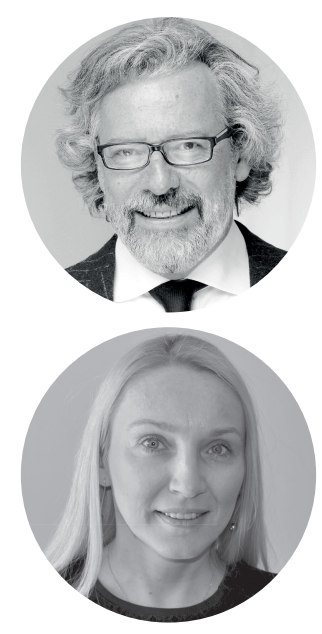

Suicide figures are declining globally. Most recent estimates suggest 788,000 deaths in 2015 as representative of the global mortality due to suicide (World Health Organization [WHO], 2017). Rates also appear to be decreasing remarkably, with older adults (65 years and over) witnessing the most relevant decrease (Bertolote \& De Leo, 2012). Reasons for this welcome phenomenon are not too clear, although general improvements in the quality of life of older citizens and ameliorated health assistance conditions are reported as the most convincing explanations (WHO, 2014). Despite these changes, older adults continue to represent the segment of population most exposed to the risk of suicide nearly everywhere in the world.

The gender paradox in suicide rates (i.e., the difference between sexes that sees a greater prevalence of fatal acts among male individuals) is often explained by the more developed skills in help-seeking behavior of female subjects, and the use of more lethal suicide methods in males (such as the use of firearms and hanging; Kõlves, McDonough, Crompton, \& De Leo, 2017; Schriivers, Bollen, \& Sabbe, 2012). In high-income countries, it is generally noted that the suicide rates ratio between males and females stands at around 3:1. In low- and middle-income countries, this ratio averages around 1.5:1 (WHO, 2014). However, with advancing age these ratios change profoundly, with rates greatly increasing among men. A study of centenarians by Shah, Zarate-Escudero, Bhat, De Leo, and Erlangsen, using data from 17 countries (those able to provide data for the oldest-olds), showed the existence of a ratio of $8: 1$ between males and females, a fact that seems to suggest the accumulation of multiple risk factors among men and an apparently better adaptation of women at such advanced age (Shah et al., 2014).

\section{Senectus Ipsa Morbus}

From a public health perspective, the rapidly aging population of many countries of the world poses several challenges. Particularly the oldest-old age segment ( $85+$ years) represents the most neglected and disadvantaged group of people, characterized by the greatest burden of noncommunicable diseases, biggest degree of frailty, and highest level of social isolation and loneliness. In regard to the latter point, as demonstrated, for example, by observations in China, the percentage of people aged 65 years and more, living alone or with their spouse only, has increased from $29 \%$ of men and $24 \%$ of women in 1990 to $37 \%$ of men and $30 \%$ of women in 2000 (Yi \& Wang, 2003). The change in household structure might have affected the health outcomes among older adults (Morita, Yamamoto, Ozaki, Tsuda, \& Tanimoto, 2017). In fact, this increase in solitary or elderly-only households has been associated with a higher prevalence of dementia and disability in activities of daily living (Li, Zhang, \& Liang, 2009; Schulz \& Sherwood, 2008). While dementia does not appear to be correlated to an increased risk of suicide (Haw, Harwood, \& Hawton, 2009), expansion of disabilities in physical functioning can severely affect the quality of life of individuals and create the grounds for a number of factors commonly associated with increased suicidality in old age. In fact, lost mobility and autonomy, dependency on others, chronic pain, lack of hope for any improvement, together with alterations in the sense of self and injured personal dignity may render the life of individuals as unacceptable and meaningless (De Leo \& Arnautovska, 2016). Accumulation of affective losses (e.g., of role and status, and multiple bereavements) can create the grounds for lessening the sense of belongingness of older adults, making them feel a burden to partners and relatives, and increasing solitude and loneliness. All these aspects have fed rationalistic views around suicide in old age, pushing one to consider suicide in late life as a legitimate exit in the face of unbear- 
able living conditions (De Leo \& Diekstra, 1990). Clearly, the piling up of several risk factors appears to increase the risk of a fatal choice (De Leo \& Arnautovska, 2016). How much this choice represents the result of a careful balancing between pros and cons, especially at very advanced age, remains unclear. Depression certainly has its role in suicidal behaviors of older adults, as it has in younger age groups; however, it is possible that its contribution to cases of suicide in old age could have been overemphasized (De Leo, Draper, Snowdon, \& Kõlves, 2013). Ageist views are still persistently present even among health professionals, who tend to consider depression as a normal feature of advanced age (De Leo, 2017; Rabheru, 2004) and in any case a common reaction to life events that older adults - frail individuals - can no longer cope with.

However, the term depression should appropriately designate only a pathological alteration; depression is not an obvious response to any type of stressor, such as sudden deterioration of physical condition or financial difficulties. Feelings of sadness, disappointment, and hopelessness are frequent travel mates throughout the life of virtually all individuals, and not necessarily symptoms of clinical depression. How to recognize, then, if a common feeling like sadness has turned into depression (i.e., a mental disorder)? In the opinion of Maj (2011), the process achieves pathological dimension when it is configurable in one of the following interpretative models: (1) When the psychological reaction is not related to any unfavorable event or appears disproportionate to it; (2) when there is a particular qualitative dimension in this reaction (e.g., it can be interpreted as a gestalt, an entity that is more than the sum of its constitutive elements); and (3) for pragmatic reasons, assuming there is a continuum of gravity ranging from ordinary sadness to clinical depression (Maj, 2011). In qualifying a condition as depression in clinical terms, many psychiatrists today tend to favor the latter interpretation.

Whatever the interpretation, from a suicide prevention perspective, patients should always be approached carefully, keeping in mind that reactions to life events vary to a great extent among individuals, and that underestimating the presence of a depression (and consequently failing to treat it) would always be dangerous. This could be particularly true in the oldest-olds, where the simultaneous contribution of many physical and psychosocial stressors could easily surpass the threshold of subjective tolerability and feed individuals' desire to escape from a situation perceived as unbearable.

As exemplified by the Story of Umberto in Turning Points (De Leo, 2010, pp. 129-138), these thresholds are often pre-established and suicide plans particularly well considered. Older adults are life experts who are able to evaluate the possible consequences of their plan very carefully. It is no coincidence that their suicide notes generally appear to be devoid of emotionality that distinguishes their messages from the ones left by younger individuals (Ho, Yip, Chiu, \& Halliday, 1998). Their notes are more pragmatic and frequently contain financial arrangements for survivors, and express the desire not to be a burden to them (Linn \& Lester, 1996). Approximately $30-40 \%$ of suicide cases among older people are accompanied by a suicide note (Neulinger \& De Leo, 2001; Salib, Cawley, \& Healey, 2002) but many older adults do not leave a farewell message simply because they no longer have anyone to address it to; in other cases, individuals may have lost or compromised the ability to write/express themselves (De Leo, Draper, \& Krysinska, 2009).

\section{Failing to Intercept Suicidal Trajectories}

When a person dies, and has attended a health-care facility a few hours before suicide, this might imply that a possible rescue operation has failed. Perhaps the latter was too difficult to conduct: Maybe there was not sufficient time to properly investigate the patient's life circumstances; maybe the patient was not able to establish a trusted communication with the doctor or was disappointed by the contact. Maybe the suicidal senior presented too complex problems to allow doctors follow their usual intervention principles. Clinicians often think they can comfortably move only along the lines for which they are culturally equipped, that is, physical and mental disorders. Management of problems like life events and loneliness, for instance, are normally considered to be the task of someone else, for example, a psychologist or a social worker. Not infrequently, within the timeframe of the referral from a specialist to another, the irreversible may happen.

On the contrary, adopting an attitude - forged by time pressure - that involves some degree of oversimplification of problems (where every emotional reaction to difficulties becomes easily attributable to depression) can promote an approach too often restricted to the mere prescription of an antidepressant drug. In this way, the appreciation of the multifactorial nature of an individual's crisis becomes too limited and the chances of successfully counteracting the complexities of a dangerous suicide progression too modest.

\section{The Gender Paradox}

At any point of the life span - including very advanced age - depression is significantly more common in women than in men (WHO, 2017). In theory, given the strong association of depression with self-inflicted death, women should be more exposed to the risk of suicide. This is an- 
other aspect of the gender paradox. In fact, females seem to be able to manage life stressors better than males do and have particularly resilient attitudes even at very advanced age (Canetto, 1995). Disparities in resilience have been explained by the different coping strategies used by men and women in periods of stress (Canetto, 1995). Men are generally less inclined to show their weaknesses and seek treatment for psychological problems (Galdas, Cheater, \& Marshall, 2005) and also appear less likely than women to adhere to therapies when initiated (Vörös, Osváth, \& Fekete, 2004).

Probably for the same reasons at the basis of the gender paradox, women of advanced age seem to be the ones also able to benefit more from the few suicide preventative programs specifically designed for older adults, such as the TeleCheck-TeleHelp network in Italy (De Leo, Dello Buono, \& Dwyer, 2002) or community-based interventions in Japan (Oyama et al., 2005). All these features are probably associated with the strong relationship between the rates of suicide of men and women of very advanced age: As mentioned, at $85+$ years of age the rates ratio is 2-3 times bigger in men (it is 3:1 for most of the life span, but 8:1 in the oldest-old; Shah et al., 2014; WHO, 2014). Rather clearly, this indicates the higher level of difficulties of men, compared with women, in facing the many challenges of life when very old.

\section{Future Strategies}

Needless to say, also in late life, suicide represents a multifactorial problem that involves many interconnected aspects. These factors vary with advancing age, sex, and culture. However, risk factors for suicide in the oldest-old have been poorly investigated. The large discrepancy existing in the rates of men and women seems to suggest the urgent need for specifically focused research investigations on older individuals' suicide (Koo, Kõlves, \& De Leo, 2017).

It is true that most national suicide prevention strategies recognize the high risk of suicide in those aged 65 years and over (WHO, 2014). Strategies aimed at this age range generally promote mental health, with particular attention to early recognition and treatment of depression. However, depression is only one of the risk factors for suicide in old age and not necessarily the most important, especially in non-Western cultures (Fleischmann \& De Leo, 2014). In any case, mental health care remains of paramount importance for suicide prevention also in late life, and access to integrated mental health services and adequate treatment and support for older people and their accompanying persons should be facilitated in every possible way (De Leo \& Arnautovska, 2016). In fact, studies on the effectiveness of mental health services for the elderly provide encouraging evidence, particularly for those services involving multidisciplinary community teams able to interact with general practitioners, social services, and specialist outpatient clinics, and especially with family members of the patient, in order to prevent interruptions in the care chain (Draper \& Low, 2004).

Prevention of suicide in older people should also concentrate on the many psychosocial factors that may characterize late stages of life (De Leo \& Arnautovska, 2016). Relocation, forced institutionalization, financial insecurity, functional and cognitive deterioration, bereavement processes, and loneliness (Cacioppo \& Cacioppo, 2014) are some of the factors that may seriously compromise the quality of life of individuals and render suicide a possible option in order to escape from an unbearable situation (Zanone-Poma et al., 2014). Given the particular nature of many suicide risk factors that accumulate in late life, community gatekeepers may be ideally positioned to identify and intercept older adults' suicidal trajectories. In light of this, training them to prevent suicide of old people can be an important strategy (Conwell, 2014).

Lower mortality rates and increased longevity, including of those with mental and physical impairments, are posing enormous public health problems and stretching governmental resources to cope with a much-expanded proportion of older adults in the general population. In 2010, 40 million US citizens were aged 65 and more; by 2040 their number is expected to double (US Census Bureau, 2016). Strategic planning for adequate quality of life to this segment of the population is urgently needed at all levels (health, financial, housing, recreational, etc.). High-income countries would face the big changes in the demographic structure of their populations much earlier than the rest of the world. Predictably, these changes would deeply affect the structure of societies and their ways of functioning, including moral values and spiritual life.

The challenge is on, and not only for suicide prevention.

\section{References}

Bertolote, J., \& De Leo, D. (2012). Global suicide mortality rates - a light at the end of the tunnel? Crisis, 33, 249-253.

Cacioppo, J. T., \& Cacioppo, S. (2014). Social relationships and health: The toxic effect of perceived social isolation. Social and Personality Psychology Compass, 8, 58-72.

Canetto, S. S. (1995). Elderly women and suicidal behavior. In S. S. Canetto \& D. Lester (Eds.), Women and suicidal behaviour. New York, NY: Springer Publishing Company.

Conwell, Y. (2014). Suicide later in life. Challenges and priorities for prevention. American Journal of Preventive Medicine, 47, S244S250.

De Leo, D. (2010). Turning points. Brisbane, Australia: Australian Academic Press. 
De Leo, D. (2017). Ageism and suicide prevention. Lancet Psychiatry. https://dx.doi.org/10.1016/S2215-0366(17)30472-8

De Leo, D., \& Arnautovska, U. (2016). Prevention and treatment of suicidality in older adults. In R. O'Connor \& J. Pirkis (Eds.), International handbook of suicide prevention. Research, policy and practice (pp. 323-345). Chichester, UK: Wiley Blackwell.

De Leo, D., Dello Buono, M., \& Dwyer, J. (2002). Suicide among the elderly: The long-term impact of a telephone support and assessment intervention in northern Italy. British Journal of Psychiatry, 181, 226-229.

De Leo, D., \& Diekstra, R. F. W. (1990). Depression and suicide in late life. Göttingen, Germany: Hogrefe \& Huber.

De Leo, D., Draper, B., \& Krysinska, K. (2009). Suicidal elderly people in clinical and community settings: Risk factors, treatment and suicide prevention. In D. Wasserman \& C. Wasserman (Eds.), Oxford textbook of suicidology and suicide prevention: A global perspective (pp. 703-719). Oxford, UK: Oxford University Press.

De Leo, D., Draper, B., Snowdon, J., \& Kõlves, K. (2013). Suicides in older adults: A case-control psychological autopsy study in Australia. Journal of Psychiatric Research, 47, 980-988.

Draper, B., \& Low, L. (2004). What is the effectiveness of old-age mental health services? Retrieved from http://www.euro.who. int/document/E83685.pdf

Fleischmann, A., \& De Leo, D. (2014). The World Health Organization's report on suicide: A fundamental step in worldwide suicide prevention. Crisis, 35, 289-291.

Galdas, P. M., Cheater, F., \& Marshall, P. (2005). Men and help-seeking behaviour: Literature review. Journal of Advanced Nursing, 49, 616-623.

Haw, C., Harwood, D., \& Hawton, K. (2009). Dementia and suicidal behaviour: A review of the literature. International Psychogeriatrics, 21, 440-453.

Ho, T. P., Yip, P. S., Chiu, C. W., \& Halliday, P. (1998). Suicide notes: What do they tell us? Acta Psychiatrica Scandinavica, 98, 467473.

Kõlves, K., McDonough, M., Crompton, D., \& De Leo, D. (2017). Choice of a suicide method: Trends and characteristics. Psychiatry Research. https://doi.org/10.1016/j.psychres.2017.11.035

Koo, Y. W., Kõlves, K., \& De Leo, D. (2017). Suicide in older adults: Differences between the young-old, middle-old, and oldest-old. International Psychogeriatrics, 17, 1297-1306. https://doi.org/ $10.1017 /$ S1041610217000618

Li, L. W., Zhang, J., \& Liang J. (2009). Health among the oldest-old in China: Which living arrangements make a difference? Social Science and Medicine, 68, 220-227.

Linn, M., \& Lester, D. (1996). Content differences in suicide notes by gender and age: Serendipitous findings. Psychological Reports, $78,370$.

Maj, M. (2011). When does depression become a mental disorder? British Journal of Psychiatry, 199, 85-86.

Morita, T., Yamamoto, K., Ozaki, A., Tsuda, K., \& Tanimoto, T. (2017). The oldest-old in China. Lancet, 390, 846-847.

Neulinger, K., \& De Leo, D. (2001). Suicide in elderly and youth population: How do they differ? In D. De Leo (Ed.), Suicide and euthanasia in older adults (pp. 137-154). Göttingen, Germany: Hogrefe \& Huber Publishers.

Oyama, H., Watanabe, N., Ono, Y., Sakashita, T., Takenoshita, Y., ... Taguchi, M. (2005). Community-based suicide prevention through group activity for the elderly successfully reduced the high suicide rate for females. Psychiatry and Clinical Neurosciences, 59, 337-344.

Rabheru, K. (2004). Special issues in the management of depression in older patients. Canadian Journal of Psychiatry, 49, 41-50.

Salib, E., Cawley, S., \& Healey, R. (2002). The significance of suicide notes in the elderly. Aging and Mental Health, 6, 186-190.

Schriivers, D. L., Bollen, J., \& Sabbe, B. G. (2012). The gender paradox in suicidal behavior and its impact on the suicidal process. Journal of Affective Disorders, 138, 19-26.

Schulz, R., \& Sherwood, P. R. (2008). Physical and mental health effects of family caregiving. American Journal of Nursing, 108, 23-27.

Shah, A., Zarate-Escudero, S., Bhat, R., De Leo, D., \& Erlangsen, A. (2014). Suicide in centenarians: The international landscape. International Psychogeriatrics, 26, 1703-1708.

US Census Bureau. (2016). National population totals tables: 2010-2016. Retrieved from https://www.census.gov/data/tables/2016

Vörös, V., Osváth, P., \& Fekete, S. (2004). Gender differences in suicidal behaviour. Neuropsychopharmacology Hungarica, 6 65-71.

World Health Organization. (2014). Preventing suicide: A global imperative. Geneva, Switzerland: Author.

World Health Organization. (2017). Depression and other common mental disorders. Global health estimates. Geneva, Switzerland: Author.

Yi, Z., \& Wang, Z. (2003). Dynamics of family and elderly living arrangements in China: New lessons learned from the 2000 census. China Review, 3, 95-119.

Zanone-Poma, S., Vicentini, S., Siviero, F., Grossi, A., Toniolo, E., Cocchio, S., ... De Leo, D. (2014). Life span history of non-fatal suicidal behaviours in a large sample of general practitioners' patients: Data from Rovigo, Northern Italy. Community Mental Health Journal, 50, 981-986.

Published online December 22, 2017

Diego De Leo, MD, PhD, DSc, FRANZCP, is Emeritus Professor of Psychiatry at Griffith University, Australia, and Director of the Slovene Centre for Suicide Research, Primorska University, Slovenia. Prof. De Leo was President of the International Association for Suicide Prevention and the International Academy for Suicide Research.

Kairi Kõlves, PhD, is Principal Research Fellow at AISRAP (joined AISRAP in 2008). She has been working in suicide research and prevention since 1998. Between 1999 and 2008, she worked at the EstonianSwedish Mental Health and Suicidology Institute in Estonia.

\section{Prof. Diego De Leo}

Griffith University

Mt. Gravatt

4122 QLD

Australia

d.deleo@griffith.edu.au 